\title{
Are curved displays ergonomically advantageous over flat displays? Some positive results from three display curvature studies on monitors and TVs
}

\author{
Sungryul Park (psr926@unist.ac.kr), Jihhyeon Yi (yjh90@unist.ac.kr), Donghee Choi \\ (choidh@unist.ac.kr), Songil Lee (songil@unist.ac.kr), Gyouhyung Kyung (ghkyung@unist.ac.kr)
}

Interaction and Experience Lab, UNIST, Ulsan, Korea, 44919

\begin{abstract}
Background: Though curved display products such as curved monitors, TVs, and smartphones are available, it seems still inconclusive whether or not curved display is ergonomically advantageous over flat display, and if so in terms of what ergonomic aspects. To examine a visual display comprehensively, three aspects, productivity, safety, and well-being, should be considered. Objective: We aimed to determine whether or not display curvature is ergonomically recommendable, especially for monitors and TVs. Methods: Overall, we examined the effect of display curvature on visual task performance, visual fatigue, and/or watching experience by conducting three different studies. In Study 1, we examined the effect of display curvature (400R, 600R, 1200R, and flat) on visual task performance and visual fatigue. A visual search task was performed on 50" multi-monitors. In Study 2, we examined the effect of display curvature (600R, 1140R, 2000R, 4000R, and flat) on visual task performance, visual fatigue, and display satisfaction. A proofreading task was performed on 27" monitors. In Study 3, we examined the effect of display curvature $(2300 \mathrm{R}, 4000 \mathrm{R}, 6000 \mathrm{R}$, and flat) on presence and display satisfaction. A TV watching task was done on 55" TVs. Results: First, curved displays increased productivity. The mean visual search accuracy and speed were both higher at the $50 " 600 \mathrm{R}$ and 1200R multi-monitor settings, and the mean proofreading speed was the fastest at the $27 " 600 \mathrm{R}$ curved monitor setting. Second, curved displays increased safety. The mean visual fatigue was lower at the 50" 600R and 1200R multi-monitor settings. However, the effect of display curvature on visual fatigue was not significant in the case of the 27" monitors. Third, curved displays improved well-being. Compared to the 55" flat TV, the mean spatial presence and engagement did not significantly decrease up to a more lateral viewing position especially when the display curvature was similar to the viewing distance. The effect of display curvature on display satisfaction was, however, not significant in Studies 2 and 3. Applications: The findings of three studies suggest that display curvature, if its level is carefully selected, can improve monitors and TVs in terms of productivity, safety, and/or well-being.
\end{abstract}

Keywords: Curved display, visual ergonomics, visual task

\section{Introduction}

Since the introduction of curved display products (e.g. monitors, TVs, smartphones, and smart watches) in the market, there have been a variety of comparative studies between curved and flat displays.

Recently, it has been shown that curved displays are advantageous over flat displays in terms of visual task performance, visual fatigue, preference, and satisfaction (Czerwinski et al. 2003; Häkkinen et al. 2008). In contrast, some negative effects of display curvature were reported in terms of legibility (Lin et al. 2009; Wang et al. 2012) and visual processing speed (Mustonen et al. 2015).

In order to evaluate new display products from the perspective of visual ergonomics (IEA 2012), three aspects of productivity, safety, and well-being should be considered. However, few studies on curved display have considered all these factors.

The purpose of the current study was to comprehensively evaluate the effects of display curvature on visual task performance, visual fatigue, and viewing experience. 


\section{Method}

Three studies on display curvature were done. In the first study, visual task performance and visual fatigue were evaluated using a visual search task on a 50" multi-monitor. In the second study, visual task performance, visual fatigue, and satisfaction were evaluated using a proofreading task on five 27" mock-up monitors. In the third study, the viewer's presence and satisfaction associated with a TV watching task were evaluated using four 55" mock-up TVs.

\subsection{Study 1}

Twenty-seven students (male: 14, female: 13) participated in Study 1. Their mean (SD) age was 20.9 (1.2) yrs. Exclusion criteria were as follows: having visual acuity $<0.8$ (Wu 2011), wearing a pair of glasses, being color blind based on the Ishihara test (Ishihara and Force 1943), or suffering from any ocular disease in the past six months.

The multi-monitor used in this experiment consisted of five display panels [5 display zones; LP171EE3, LG, Korea] of $244 \mathrm{~mm}$ (width) $\times 382 \mathrm{~mm}$ (height), and the overall size was 50 " (1220 mm $\times 382 \mathrm{~mm})$. The horizontal viewing distance to the central display $\left(\mathrm{Z}_{3}\right.$; Figure 1$)$ was $500 \mathrm{~mm}$.

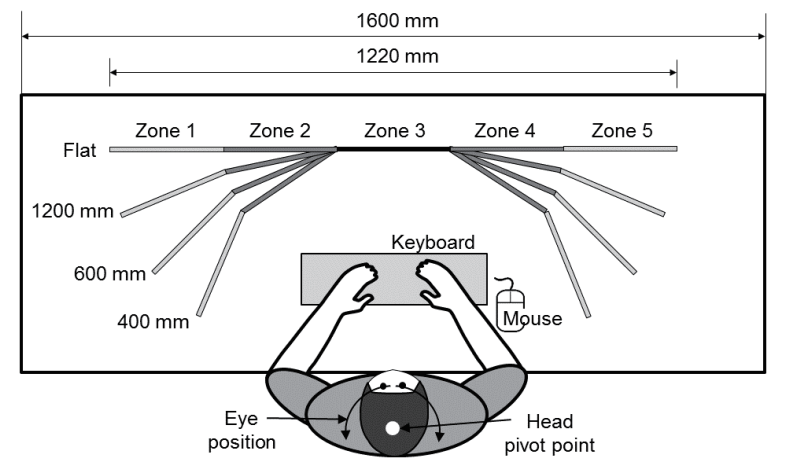

Figure 1. Experimental setting for Study 1

A pseudo text was randomly presented on a display zone for $3 \mathrm{~min}$, and a visual search task (ISO, 2008) was repeated twice on each display zone.

Two independent variables were used in Study 1. Four levels of display curvature (400R, 600R, 1200R, and flat; within-subjects) were considered. Display zones (within-subjects) had five levels $-Z_{1}$ (leftmost), $Z_{2}, Z_{3}$ (Center), $Z_{4}$, and $Z_{5}$ (rightmost). The order of presenting the display curvature was determined by using a $4 \times 4$ Latin Square.

Five dependent variables were used in Study 1. Error rate and searching speed were used to measure the visual search task performance. To measure visual fatigue, a $100 \mathrm{~mm}$ visual analogue scale (VAS), eye-complaint questionnaire [(ECQ; Steenstra et al. (2009)], and critical fusion frequency (CFF) were used.

\subsection{Study 2}

Fifty students (male: 17, female: 33) participated in Study 2. Their mean (SD) age was 22.3 (1.5) yrs. Exclusion criteria were the same as in Study 1.

The mock-up monitor used a projected image (EB-4950WU, EPSON) on a 27" polycarbonate rear screen $(603 \mathrm{~mm} \times 346 \mathrm{~mm}$; Exzen, Korea; Figure 2). Image distortion on the screen was corrected using Desktop Warpalizer ${ }^{\circledR}$ (UniVisual Technologies, Sweden). The viewing distance used in Study 2 was $600 \mathrm{~mm}$.

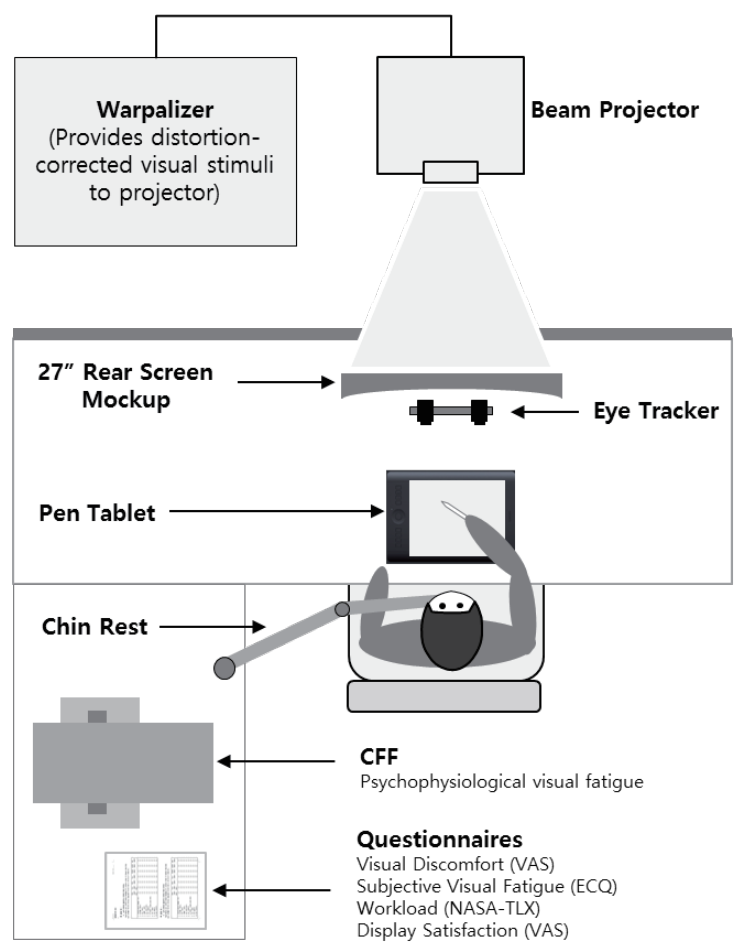

Figure 2. Experimental setting for Study 2

Each participant performed a proofreading task on a screen with a specific curvature. Korean texts from the Naver Cast (http://navercast.naver.com/) were used for proofreading. A reference article without grammatical errors was displayed on the left side of the screen, and an article with grammatical errors on the right side. Referring to the left article, each participant needed to 
find and mark errors in the article on the right side using editing symbols.

Two independent variables were used in Study 2. Five levels of display curvature (600R, 1140R, 2000R, 4000R, and flat; between-subjects) were considered. Task durations (five levels; within-subjects) were 0,15 , 30,45 , and $60 \mathrm{~min}$. Nine dependent variables were used in Study 2. Proofreading speed and accuracy, visual discomfort, subjective visual fatigue, psychophysiological visual fatigue, blink duration, blink frequency, mental workload, and display satisfaction were measured.

\subsection{Study 3}

Fifty-six students (male: 20, female: 36) participated in Study 3. Their mean (SD) age was 20.9 (1.5) yrs. Exclusion criteria for participation were the same as in Study 1. Each mock-up TV screen used in the experiment was made by attaching a projection film (Sunnano, Korea) on the front of a $55^{\prime \prime}(1218 \mathrm{~mm} \times 685 \mathrm{~mm})$ styrofoam panel having a specific curvature. Images were projected on the screen (Figure 3).

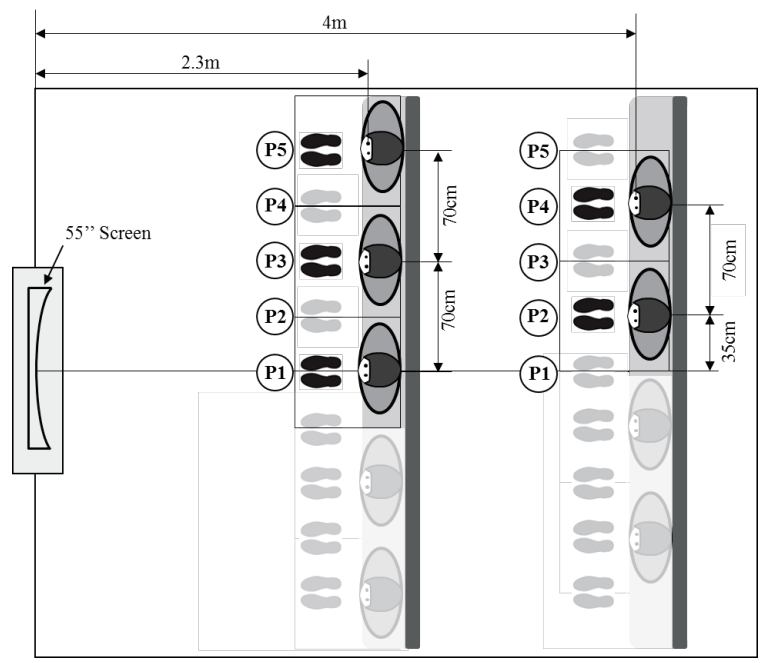

Figure 3. Experimental setting for Study 3

The visual stimuli used in Study 3 consisted of ten 5-min videos, which was a combination of 1-min sample from five types of video. One of ten videos was randomly used for each viewing distance $\times$ viewing location condition. Image distortions on the screen were corrected using Desktop

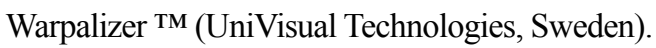

Three independent variables were used in Study 3. Four levels of display curvature $(2300 \mathrm{R}, 4000 \mathrm{R}, 6000 \mathrm{R}$, and flat; between-subjects) were considered. Viewing distance had two levels (2.3m and 4m; within-subjects). Lateral viewing position (within-subjects) had five levels $-\mathrm{P}_{1}$ (Center), $\mathrm{P}_{2}$ (35cm rightward from $\left.\mathrm{P}_{1}\right), \mathrm{P}_{3}(70 \mathrm{~cm}), \mathrm{P}_{4}(105 \mathrm{~cm})$, and $\mathrm{P}_{5}$ $(140 \mathrm{~cm})$. Seven dependent variables related to watching experience were used in Study 3 - presence, visual comfort, image quality, and display satisfaction. Presence was further divided into four categories - spatial presence, engagement, ecological validity, and negative effects.

\section{Results and discussion}

\subsection{Study 1}

In the 50" multi-monitor study, curved display settings showed a better visual search task performance than the flat display setting. Compared to the flat setting, the mean error rate was $20 \%$ lower at the $1200 \mathrm{R}$ setting, and the mean speed was $8 \%$ faster at the $400 \mathrm{R}$ and $600 \mathrm{R}$ settings. Task performance was degraded as the display became planar, and the display zone was more lateral. The mean error rate was not significantly different across the display zones of the curved display settings, while it increased by $37 \%$ at $Z_{1}$ (leftmost) compared to $Z_{3}$ (center) of the flat display setting. The mean speed was not significantly different across the display zones in the case of the $400 \mathrm{R}$ and $600 \mathrm{R}$ settings, while it decreased by $10 \%-18 \%$ at $Z_{1}$ and $Z_{5}$ (the left and rightmost) compared to $Z_{3}$ in the case of the $1200 \mathrm{R}$ and flat settings. The mean error rate increased by $8 \%$ and the mean search speed decreased by $2 \%$ during the second session, compared to the first session.

Less visual fatigue was reported at the curved display settings than at the flat display setting. The mean perceived visual fatigue was $13 \%$ lower at the $600 \mathrm{R}$ setting than at the flat setting. In addition, in the case of the curved display setting, visual fatigue was similar across the display zones, while it increased up to $45 \%$ at $Z_{1}$ compared to $Z_{3}$ in the case of the flat setting.

\subsection{Study 2}

The effect of display curvature on proofreading speed on the 27 " monitors was significant. The mean proofreading speed was $14 \%$ faster at the $600 \mathrm{R}$ than at the flat. The effect of task duration on proofreading speed and accuracy was 
significant. During the 60-minute task, the mean proofreading speed increased by $16 \%$, while the proofreading accuracy decreased by $22 \%$.

The effects of display curvature on visual discomfort, visual fatigue, mental workload, and display satisfaction were not significant, while the effect of task duration was significant. During the 60-min proofreading task, the mean visual discomfort increased by $207 \%$, the mean perceived visual fatigue increased by $166 \%$, the mean CFF decreased by $0.6 \mathrm{~Hz}$ (indicating an increase in visual fatigue), and the mean mental workload increased by $153 \%$.

\subsection{Study 3}

For the 55" TVs, the mean spatial presence and engagement were high when the display curvature was equal to the viewing distance, and began to decrease at a more lateral viewing position. Compared to the $4000 \mathrm{R}-4 \mathrm{~m}-\mathrm{P}_{1}$, which provided the highest mean spatial presence, the mean spatial presence at the $2300 \mathrm{R}, 4000 \mathrm{R}$, and $6000 \mathrm{R}$ settings at the viewing distance of $2.3 \mathrm{~m}$ reduced by $30 \%-39 \%$ at $\mathrm{P}_{5}$, but the mean spatial presence at the flat setting at the same distance began to reduce by $32 \%-37 \%$ at $\mathrm{P}_{3}$. In addition, the mean spatial presence at the $4000 \mathrm{R}$ setting at the viewing distance of $4 \mathrm{~m}$ reduced by $32 \%$ at $\mathrm{P}_{5}$, while those of the 2300R and flat settings reduced by $32 \%$ (at $\mathrm{P}_{4}$ ) and $31 \%-44 \%$ (at $\mathrm{P}_{1}, \mathrm{P}_{3}, \mathrm{P}_{4}$, and $\mathrm{P}_{5}$ ), respectively. Compared to the $4000 \mathrm{R}-4 \mathrm{~m}-\mathrm{P}_{1}$, which provided the highest mean engagement, the mean engagement at the curved settings (2300R, 4000R, and 6000R) at the viewing distance of $2.3 \mathrm{~m}$ decreased by $22 \%-27 \%$ at $\mathrm{P}_{5}$, but that at the flat setting decreased by $30 \%$ at $\mathrm{P}_{3}-\mathrm{P}_{5}$. In addition, the mean engagement at the $4000 \mathrm{R}$ and $6000 \mathrm{R}$ settings at the viewing distance of $4 \mathrm{~m}$ decreased by $29 \%$ and $24 \%$ at $\mathrm{P}_{5}$, while those at the $2300 \mathrm{R}$ and flat settings decreased up to $30 \%$ at $\mathrm{P}_{3}-\mathrm{P}_{5}$. For ecological validity, negative effects, visual comfort, image quality, and display satisfaction, no effect was significant.

\section{Conclusions}

Three studies examined the effects of display curvature on user productivity (visual task performance), safety (visual fatigue and visual discomfort), and well-being (presence and satisfaction). The results of these three studies can contribute to determining appropriate display curvatures for monitors or TVs.

\section{Acknowledgements}

This research was supported by the Basic Science Research Program through the National Research Foundation of Korea funded by the Ministry of Education (NRF-2016R1A2B4010158).

\section{References}

Czerwinski, M. et al., 2003. "Toward Characterizing the Productivity Benefits of Very Large Displays." In Proceedings of INTERACT 9-16.

Häkkinen, J. et al., 2008. "Reading Experience with Curved Hand-Held Displays." Journal of the Society for Information Display, 16 (11), 1099.

Ishihara, S. and Force, R.a.A., 1943. Ishihara Tests for Colour Blindness: Sydney: Shephard \& Newman.

ISO, 2008. Ergonomics of Human-System Interaction Part 304: User Performance Test Methods for Electronic Visual Displays.

Lin, Y.T. et al., 2009. "Investigation of Legibility and Visual Fatigue for Simulated Flexible Electronic Paper under Various Surface Treatments and Ambient Illumination Conditions." Applied Ergonomics, 40 (5), 922-8.

Mustonen, T. et al., 2015. "Visual Performance with Small Concave and Convex Displays." Human Factors: The Journal of the Human Factors and Ergonomics Society.

Steenstra, I.A., Sluiter, J.K., and Frings-Dresen, M.H., 2009. "The Eye-Complaint Questionnaire in a Visual Display Unit Work Environment: Internal Consistency and Test-Retest Reliability." Ergonomics, 52 (3), 334-44.

Wang, A.H., Hwang, S.L., and Kuo, H.T., 2012. "Effects of Bending Curvature and Ambient Illuminance on the Visual Performance of young and elderly participants Using Simulated Electronic Paper Displays." Displays, 33 (1), 36-41.

Wu, H.C., 2011. "Electronic Paper Display Preferred Viewing Distance and Character Size for Different Age Groups." Ergonomics, 54 (9), 806-14.

http://www.iea.cc/index.php 